PPPL-3156, Preprint: March 1996, UC-420, 427

\title{
Role of Flow Shear in Enhanced Core Confinement Regimes
}

T.S. Hahm and K.H. Burrell

The importance of the ExB flow shear in various enhanced confinement regimes is discussed in terms of the turbulence suppression criterion in toroidal geometry. This criterion is then further generalized to include the poloidal angle dependence of the equilibrium electrostatic potential. The implication of the recently observed in-out asymmetry in the fluctuation behavior in DIII-D VH-mode is discussed.

\author{
RECEIVED \\ MAR 251996 \\ O STI
}

\section{DISCLAIMER}

This report was prepared as an account of work sponsored by an agency of the United States Government. Neither the United States Government nor any agency thereof, nor any of their employees, makes any warranty, express or implied, or assumes any legal liability or responsibility for the accuracy, completeness, or usefulness of any information, apparatus, product, or process disclosed, or represents that its use would not infringe privately owned rights. Reference herein to any specific commercial product, process, or service by trade name, trademark, manufacturer, or otherwise does not necessarily constitute or imply its endorsement, recommendation, or favoring by the United States Government or any agency thereof. The views and opinions of authors expressed herein do not necessarily state or reflect those of the United States Government or any agency thereof. 


\title{
Role of Flow Shear in Enhanced Core Confinement Regimes
}

\author{
T S Hahm ${ }^{1}$ and K H Burrell ${ }^{2}$
}

${ }^{1}$ Princeton Plasma Physics Laboratory, P.O. Box 451, Princeton, NJ 08543

${ }^{2}$ General Atomics, P.O. Box 85608, San Diego, CA92186-9784

\begin{abstract}
The importance of the $\mathbf{E} \times \mathbf{B}$ flow shear in various enhanced confinement regimes is discussed in terms of the turbulence suppression criterion in toroidal geometry. This criterion is then further generalized to include the poloidal angle dependence of the equilibrium electrostatic potential. The implication of the recently observed in-out asymmetry in the fluctuation behavior in DIII-D VH-mode is discussed.
\end{abstract}

\section{Introduction}

It is well known that the $\mathbf{E} \times \mathbf{B}$ flow shear plays a crucial role in the $\mathrm{L}$ to $\mathrm{H}$ transition of many tokamak plasmas[1-4]. In this paper, the importance of $\mathbf{E} \times \mathbf{B}$ flow shear in various enhanced core confinement regimes is discussed. Our recent nonlinear theory of flow shear induced fluctuation suppression in an arbitrary shape finite aspect ratio tokamak plasma[5] indicates that the radial shear of $E_{r}^{(0)} / R B_{\theta}$ is the quantity of interest. This result has compared well with magnetic braking experiment on VH-mode in DIII-D [6]. While this criterion can be applied universally to various enhanced confinement regimes in tokamaks $[1,2,6]$, their dominant contributors to this term vary. A possible origin of this term can be plasma rotation due to unbalanced neutral beam injection (such as in the DIII-D VH-mode) or ion Bernstein waves (PBX-M CH-mode[7]), as well as peaked ion pressure profiles (TFTR Supershot[8]). Furthermore, from the explicit dependence on $B_{\theta}$, we can 
gain new insight into the confinement improvement in reversed shear configurations $[9,10]$.

In Sec. III, our previous fluctuation suppression criterion in toroidal geometry $[5,11]$ is further generalized to include the poloidal angle dependent part of the equilibrium electrostatic potential which can be produced by a strong toroidal rotation of low density plasmas[12, 13]. Finally, a recently observed in-out asymmetry in the fluctuation behavior in DIII-D VH-mode plasma[14] is discussed in Sec. IV.

\section{Role of $\mathbf{E} \times \mathbf{B}$ Flow Shear in Enhanced Core Confinement Regimes}

In our previous work[5], it has been shown that the fluctuation suppression occurs when the decorrelation rate of the ambient turbulence $\Delta \omega_{T}$ is exceeded by the following shearing rate $\omega_{s}$ in toroidal geometry, $\omega_{s} \equiv\left(\frac{\Delta \psi_{0}}{\Delta \phi}\right)\left|\frac{\partial^{2}}{\partial \psi^{2}} \Phi_{0}(\psi)\right|=\left(\frac{\Delta \psi_{0}}{\Delta \phi}\right)\left|\frac{\partial}{\partial \psi}\left(\frac{E_{r}^{(0)}}{R B_{\theta}}\right)\right|$. Here, $\Delta r_{0} \equiv \Delta \psi_{0} / R B_{\theta}$ and $R \Delta \phi$ are the correlation lengths in the radial and toroidal directions respectively. The radial force balance equation relates $E_{r}^{(0)}$ to plasma rotation velocity and the pressure gradient, $E_{r}^{(0)}=u_{\phi} B_{\theta}-u_{\theta} B_{\phi}+\frac{1}{n_{i} e_{i}} \frac{\partial}{\partial r} P_{i}$. Straightforward manipulation of this relation leads to an expression which exhibits various contributors to the quantity of interest, the radial shear of $E_{r}^{(0)} / R B_{\theta}$;

$$
\frac{\partial}{\partial \psi}\left(\frac{E_{r}^{(0)}}{R B_{\theta}}\right)=\frac{\partial}{\partial \psi}\left(\frac{u_{\phi}}{R}\right)-\frac{\partial}{\partial \psi}\left(\frac{u_{\theta} B_{\phi}}{R B_{\theta}}\right)+\frac{1}{e_{i}} \frac{\partial}{\partial \psi} \frac{1}{n_{i}} \frac{\partial}{\partial \psi}\left(n_{i} T_{i}\right)
$$

In the following examples, there is evidence that $\mathbf{E} \times \mathbf{B}$ flow shear plays a crucial role in the confinement improvement. We indicate the relative importance of each term on the right hand side for various enhanced confinement regimes.

(i) $\mathrm{H}$-mode in DIII-D; In the plasma edge across the $\mathrm{L}$ to $\mathrm{H}$ transition, the ion poloidal flow and the pressure gradient terms are both important. While the initial change in $E_{r}$ is believed to be due to a change in $u_{\theta}$, most of the fluctuation suppression occurs in the later phase in which the pressure gradient term becomes important $[2,6]$.

(ii) VH-mode in DIII-D; Due to broad $E_{r}$ shear layer, the toroidal theory specific geometric factors in Eq.(1) are quite important. Magnetic braking experiments on VH mode in DIII-D indeed show that there is a clear reduction in the thermal diffusivity in the same region where the shear in $E_{r} / R B_{\theta}$ has changed. Most contribution to $\frac{\partial}{\partial \psi}\left(\frac{E_{r}^{(0)}}{R B_{\theta}}\right)$ comes from the toroidal flow contribution (the first term on the RHS)[6]. 
(iii) CH-mode in PBX-M; Location of the internal transport barrier is consistent with the ion Bernstein wave induced ponderomotive force driven poloidal shear flow model $[7,15]$.

(iv) Supershot in TFTR; Reduction of radial correlation length with increasing toroidal velocity has been observed[16] in qualitative agreement with the prediction of the two point nonlinear theory in toroidal geometry $[5,11]$. The region of sharp gradient of $u_{\phi}$ approximately corresponds to that of $T_{i}[17]$. It is interesting to observe that for co-NBI, the toroidal flow term and the last term on the RHS add, making the fluctuation suppression easier. The last term can be written as a sum of the following two terms which are both negative for the typical peaked profiles at tokamak plasma core,

$$
-\frac{1}{n_{i}^{2}}\left(\frac{\partial n_{i}}{\partial \psi}\right) \frac{\partial}{\partial \psi}\left(n_{i} T_{i}\right)+\frac{1}{n_{i}} \frac{\partial^{2}}{\partial \psi^{2}}\left(n_{i} T_{i}\right)
$$

(v) Enhanced Reversed Shear Modes in TFTR and DIII-D; Although more quantitative data analyses are needed to confirm the importance of $\mathbf{E} \times \mathbf{B}$ flow shear, aforementioned observation regarding the signs of toroidal flow term and the last term also applies to this case. Furthermore, the $B_{\theta}$ dependence contained in the definition of the poloidal flux $\left(\frac{\partial}{\partial \psi} \equiv\right.$ $\left.\frac{1}{R B_{\theta}} \frac{\partial}{\partial r}\right)$ tends to enhance the relative importance of the pressure and density gradient terms (the last term on the RHS of Eq.(1)) in comparison to the flow terms in the reversed shear region. Therefore, it is essential that this expression in toroidal geometry must be used in comparison to the experimental data to properly address the physics origin of the enhanced confinement in reversed shear mode[9, 10].

\section{Effects of Poloidal Angle Dependent Electrostatic Potential}

In our previous works[1,5], $\Phi^{(0)}$ has been assumed to be a flux function. This simplifying assumption needs to be improved when there is strong toroidal plasma rotation. In this case, $\Phi^{(0)}$ depends on the poloidal angle $\theta$ also and the two point nonlinear analysis becomes more involved. A crucial modification comes through the introduction of $\theta$-dependence of $E_{r}^{(0)}$ as well as $E_{\theta}^{(0)}$. Now, $V_{E}^{(0)}$ has a radial component as well as the usual (non radial) perpendicular component. The radial and poloidal variations of each component must be included in the two point nonlinear analysis. The two-point correlation evolution equation 
is then derived following the standard procedure,

$$
\left\{\frac{\partial}{\partial t}+\left(\psi_{-} \Omega_{\psi \psi}+\eta_{-} \Omega_{\theta \psi}\right) \frac{\partial}{\partial \phi_{-}}-\left(\psi_{-} \Omega_{\psi \theta}+\eta_{-} \Omega_{\theta \theta}\right) \frac{\partial}{\partial \psi_{-}}-D_{-}^{\text {eff }} \frac{\partial^{2}}{\partial \phi_{-}^{2}}\right\}<\delta H(1) \delta H(2)>=S_{2} .
$$

Here, the radial shear and poloidal variation of the angular rotation frequency in perpendicular direction are given by $\Omega_{\psi \psi} \equiv-\frac{\partial^{2}}{\partial \psi^{2}} \Phi^{(0)}(\psi, \theta)$ and $\Omega_{\theta \psi} \equiv-\frac{\partial^{2}}{\partial \theta \partial \psi} \Phi^{(0)}(\psi, \theta)$. The radial shear and poloidal variation of the radial $\mathbf{E} \times \mathbf{B}$ velocity are given by $\Omega_{\psi \theta} \equiv \frac{\partial}{\partial \psi}\left(\frac{1}{\nu} \frac{\partial}{\partial \theta} \Phi^{(0)}(\psi, \theta)\right)$ and $\Omega_{\theta \theta} \equiv \frac{\partial}{\partial \theta}\left(\frac{1}{\nu} \frac{\partial}{\partial \theta} \Phi^{(0)}(\psi, \theta)\right)$. Here, $\nu$ is the local magnetic field pitch. Other notations are standard and have been defined in Hahm and Burrell[5]. The results of two point nonlinear analysis indicate that fluctuation suppression occurs when the following inequality involving the decorrelation rate of the ambient turbulence $\Delta \omega_{T}$ and the shearing rates, $\omega_{s}$ and $\Omega_{\psi \theta}$ is satisfied;

$$
\omega_{s}^{2} \equiv\left(\frac{\Delta \psi_{0}}{\Delta \phi}\right)^{2}\left|\frac{\partial^{2}}{\partial \psi^{2}} \Phi^{(0)}(\psi, \theta)\right|^{2}>\Delta \omega_{T}\left(\Delta \omega_{T}+\Omega_{\psi \theta}\right)
$$

We observe that $\Omega_{\psi \theta}$ vanishes at the mid-plane where the fluctuations are typically measured. Therefore, our previous criterion for the fluctuation suppression[5] can still be applied there. $\Omega_{\theta \psi}$ and $\Omega_{\theta \theta}$ do not affect the fluctuation level directly, although they distort the shape of the eddies.

\section{In-Out Asymmetry in Fluctuation Suppression}

The investigation of in-out asymmetries in fluctuation suppression not only imposes a stringent test on the hypothesis of flow shear induced suppression of turbulence, but also provides useful information on the nature of ambient turbulence. Recent results from the heterodyne FIR scattering measurements of density fluctuations in DIII-D VH-modes with magnetic braking[14] have indicated the following interesting features.

(i)The spectral intensity which originates from the strongly rotating VH-mode plasma core is feeble. This suggests that the core turbulence is suppressed due to the $\mathbf{E} \times \mathbf{B}$ flow shear.

(ii) After the magnetic braking is applied, lowering the plasma rotation, the broadband turbulence associated with the core returns. Interestingly, the spectral range of turbulence which initially returns is associated with the portion of the core on the high field side (in- 
side). To be compatible with this observation, the flow shear induced turbulence suppression criterion presented in Eq.(3) must have been violated at the inside first.

(iii) After the plasma slows down considerably, the intensity which originates from the low field side (outside) is higher than that from inside. This implies a ballooning-like character of the ambient turbulence with higher amplitude at the outside.

Since the ambient turbulence is stronger at the outside as observed in phase (iii), the shearing rate must be significantly higher at the outside to explain the feature in phase (ii). As discussed in Ref. 5, since density fluctuation measurements via microwave scattering are performed for a number of specific values of $k_{\theta}$ which are determined by the scattering geometry, it is more useful to write $\omega_{s}$ in terms of $\Delta \theta=\Delta \phi / \nu$ and $\Delta r_{0} ; \omega_{s}=\left(\frac{R B_{\theta} \Delta r_{0}}{\nu \Delta \theta}\right)\left|\frac{\partial^{2}}{\partial \psi^{2}} \Phi^{(0)}(\psi)\right|$. Then, for fixed $\Delta \theta, \omega_{s}$ varies like $R B_{\theta} / \nu \simeq\left(R B_{\theta}\right)^{2} / r B_{\phi} \propto R^{3}$, and the shearing rate is significantly higher at the large major radius side. We recall that the origin of the major radius dependence is flux expansion $\left(\Delta \psi=R B_{\theta} \Delta r\right)$ and the variation of the local magnetic pitch $\nu$. Another possible origin of the poloidal angle dependence in the shearing rate is strong plasma toroidal rotation $[12,13]$ as discussed in Sec.III. In this case,

$$
\Omega_{\psi \psi} \simeq-\frac{\partial^{2}}{\partial \psi^{2}} \Phi_{0}(\psi)+\frac{M_{i} R_{0} \cos \theta}{e_{i}} \frac{\partial^{2}}{\partial \psi^{2}}\left[\frac{T_{e}}{T_{i}+T_{e}} r \omega(\psi)^{2}\right]
$$

where $\omega_{s} \equiv\left(\frac{\Delta \psi_{0}}{\Delta \phi}\right)\left|\Omega_{\psi \psi}\right|$, and $\omega(\psi)$ is the angular frequency of the plasma toroidal rotation.

In conclusion, $\mathbf{E} \times \mathbf{B}$ flow shear induced suppression of turbulence still remains the best hypothesis for the confinement improvement even after the emergence of various core enhanced confinement regimes and more sophisticated fluctuation measurements.

\section{Acknowledgments}

The authors would like to thank P.H. Diamond, F.L. Hinton, Y.B. Kim, C.E. Bush, S. M. Kaye, M. Ono, and C. Rettig for useful discussions. They are also grateful to W.M. Tang and V. Chan for encouragement. This work was supported by the U.S. Department of Energy Contract No. DE-AC02-76-CHO-3073 and the U.S. Department of Energy Contract No. DE-AC03-89ER51114. 


\section{References}

[1] Burrell K H et al 1992 Plasma Phys. Contr. Fusion 341859

[2] Burrell K H et al 1994 Phys. Plasmas 11536

[3] Biglari H, Diamond P H, and Terry P W 1990 Phys. Fluids B 21

[4] Shaing K C et al 1990 Phys. Fluids B 21492

[5] Hahm T S and Burrell K H 1995 Phys. Plasmas 21648

[6] Burrell K H et al 1995 Plasma Physics and Controlled Nuclear Fusion Research 1994 (in press)

[7] Ono M et al 1995 Plasma Physics and Controlled Nuclear Fusion Research 1994 (in press)

[8] Zarnstorff M C et al 1989 Plasma Physics and Controlled Nuclear Fusion Research 1988 Vol 2 (International Atomic Energy Agency, Vienna) p 183

[9] Levinton F M et al 1995 submitted for publication in Phys. Rev. Lett.

[10] Strait E J et al 1995 submitted for publication in Phys. Rev. Lett.

[11] Hahm T S 1994 Phys. Plasma 12940

[12] Hinton F L and Wong S K 1985 Phys. Fluids 283082

[13] Connor J W et al 1987 Plasma Phys. Contr. Fusion 29919

[14] Rettig C L et al 1995 Private communications

[15] Craddock G G et al 1994 Phys. Plasmas 11944

[16] Paul S F et al 1995 Private Communications

[17] Bush C E 1995 Private Communications 